\title{
Maximal subsets free of arithmetic progressions in arbitrary sets *
}

\author{
Semchankau Aliaksei
}

March 4, 2022

\begin{abstract}
We consider the problem of determining the maximum cardinality of a subset containing no arithmetic progressions of length $k$ in a given set of size $n$. It is proved that it is sufficient, in a certain sense, to consider the interval $[1, \ldots, n]$. The study continues the work of Komlós, Sulyok, and Szemerédi.
\end{abstract}

\section{Introduction}

Let us consider an arbitrary set $B \subseteq \mathbb{Z}$ and integer $k \geqslant 3$. We define the value $f_{k}(B)$ to be the cardinality of maximal subset of $B$, which does not contan nontrivial arithmetical progression of length $k$ (we say arithmetical progression is trivial if all of its elements are equal). Let us consider the function

$$
\phi_{k}(n):=\min _{|B|=n} f_{k}(B) .
$$

Now we introduce the function $g_{k}(n):=f_{k}([1,2, \ldots, n])$. Let $\rho_{k}(n):=g_{k}(n) / n$ be a density of maximal set free of arithmetical progressions of length $k$ in segment $[1, \ldots, n]$. We know following estimates for $\rho_{k}(n)$ :

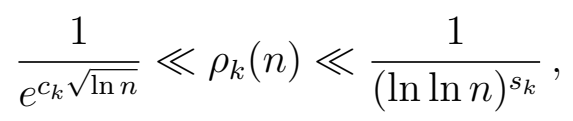

where $c_{k}, s_{k}$ are positive constants, depending only on $k$. Lower bound belongs to Behrend [Beh46], and upper bound belongs to Gowers [Gow01]. Historical retrospective on special case $k=3$ can be found in works [Shk06], [Blo12].

At first sight it seems natural to expect the equality $\phi_{k}(n)=g_{k}(n)$ to hold, although it turns out to be false already for $n=5, k=3: g_{3}(5)=f_{3}([1,2,3,4,5])=4>3=f_{3}(\{1,2,3,4,7\})=\phi_{3}(5)$. However, intuition still predicts that $\phi_{k}(n)$ does not differ much from $g_{k}(n)$. In this direction it was proved by Komlós, Sulyok, and Szemerédi [KSS75] that following inequality holds:

$$
\phi_{3}(n)>\left(1 / 2^{15}+o(1)\right) g_{3}(n), n \rightarrow \infty .
$$

*This work is supported by the Russian Science Foundation under grant 14-11-00433. 
In O'Bryent's work [OBr13] it is stated, without proof, that constant $1 / 2^{15}$ might be improved to $1 / 34$.

In here we demonstrate the following:

Theorem 1. For any integer $k \geqslant 3$ there exists such sequence $n_{1}<n_{2}<\ldots$ of natural numbers such that for any element $n$ in it following inequality holds:

$$
\phi_{k}(n)>(1 / 4+o(1)) g_{k}(n) .
$$

Furthermore, the sequence $n_{1}<n_{2}<\ldots$ is rather dense in the sense that any segment of the form $\left[n, n e^{\left.(\ln n)^{1 / 2+o(1)}\right]}\right.$ contains at least one element of this sequence.

As we see this result improves bound from [KSS75] for a subsequence of $\mathbb{N}$. We obtain constant $1 / 4$ since we 'compress' given set of numbers modulo a prime number twice and keep roughly half of the elements each time. Our method differs from the one presented at [KSS75] by fewer amount of operations (constists of 2 'compressions'), and therefore by saving more elements of the initial set.

For natural $n$ we denote by $[n]$ the segment $[1, \ldots, n]$.

\section{Compressing Lemmas}

Let us consider some set of integers $X=\left\{x_{1}, x_{2}, \cdots, x_{n}\right\}$. We call set $Y=\left\{y_{1}, y_{2}, \ldots, \cdots, y_{n}\right\}$ a compression of set $X$, if for any triples $(i, j, k) \in[n]^{3}$ equality $x_{i}-2 x_{j}+x_{k}=0$ implies $y_{i}-2 y_{j}+y_{k}=0$ (notice that we do not imply any order of $x_{i}$ and $y_{i}$ ). This definition is closely related to Freiman homomorphism, see [TV06].

Now we state a hypothesis, which we prove only in special case, which however would suffice for us.

Hypothesis 1. For any $\epsilon>0$ there is such subpolynomial function $h(n)=h_{\epsilon}(n)$, such that for any integer set $X$ of size $n$ there exists such $Y \subseteq X,|Y| \leqslant \epsilon n$, for which $X \backslash Y$ might be compressed into subset of segment $[n h(n)]$.

We prove it for all $\epsilon \in(3 / 4,1)$. For the sake of transparency, we break the proof into several lemmas. Since we are only interested in behaviour of $h(n)$ for large $n$, we would only consider a case when $n$ is large enough.

Lemma 2.1 (on compression into an interval of exponential length). Any set of size $n$ might be compressed into a subset of the segment $\left[4 n^{4} 6^{n / 2}\right]$.

Proof. Having set $X$ we want to build $Y \subset\left[4 n^{4} 6^{n / 2}\right]$ such that $Y$ is a compression of $X$.

We assign to $X$ a following matrix $A$. Let us enumerate all nontrivial arithmetical progressions of length 3 in $X$ :

$$
\left(i_{1}, j_{1}, k_{1}\right),\left(i_{2}, j_{2}, k_{2}\right), \cdots,\left(i_{p}, j_{p}, k_{p}\right)
$$

where $p$ is the total amount of progressions. Clearly, for any triple $\left(i_{s}, j_{s}, k_{s}\right)$ equality

$$
x_{i_{s}}-2 x_{j_{s}}+x_{k_{s}}=0
$$

holds. We set $A$ to be a matrix of size $p \times n$. At $s$ th row of $A$ we put 1 at $i_{s}$ th and $k_{s}$ th column, and -2 at $j_{s}$ th column. Other entries are occupied with zeros. 
For example, set $X=\{1,2,3,4,5\}$ would be assigned with the following matrix:

$$
A=\left(\begin{array}{ccccc}
1 & -2 & 1 & 0 & 0 \\
0 & 1 & -2 & 1 & 0 \\
0 & 0 & 1 & -2 & 1 \\
1 & 0 & -2 & 0 & 1
\end{array}\right)
$$

It is clear from the definition of matrix $A$ that

$$
A\left(\begin{array}{c}
x_{1} \\
x_{2} \\
\cdots \\
x_{n}
\end{array}\right)=\left(\begin{array}{c}
0 \\
0 \\
\cdots \\
0
\end{array}\right)
$$

Furthermore, $Y$ is a compression of $X$ if and only if

$$
A\left(\begin{array}{c}
y_{1} \\
y_{2} \\
\cdots \\
y_{n}
\end{array}\right)=\left(\begin{array}{c}
0 \\
0 \\
\cdots \\
0
\end{array}\right)
$$

Let us consider an arbitrary set $X$ of size $n$ and its assigned matrix $A$ : $A x=0$, where $x=$ $\left(x_{1}, \cdots, x_{n}\right)^{T}$. We would demonstrate the existion of such $y=\left(y_{1}, \ldots, y_{n}\right)$ such that its coordinates are distinct natural numbers not exceeding $4 n^{4} 6^{n / 2}$, satisfying $A y=0$.

Let us solve the equation $A x=0$. We choose maximal amount of linearly independent rows and put them to new matrix $A^{\prime}$. Certainly, $A^{\prime} x=0 \Leftrightarrow A x=0$.

We denote size of $A^{\prime}$ by $m \times n, m<n$ (clearly $A$ and $A^{\prime}$ are degenerate, since sum of elements in each row equals 0 ). Let us distinguish independent (basis) variables from dependent ones. Clearly, there are exactly $m$ dependent variables among $x_{1}, x_{2}, \ldots, x_{n}$. Let us swap coordinates in $x=\left(x_{1}, \ldots, x_{n}\right)$ and rows in $A^{\prime}$ in such a way such that first coordinates of $x$ are dependent, and last coordinates are independent. Via the Gauss elimination method we reduce the system to the following form:

$$
A^{\prime \prime} x=\left(\begin{array}{cccc}
1 & 0 & 0 & \ldots \\
0 & 1 & 0 & \ldots \\
0 & 0 & 1 & \ldots
\end{array}\right)\left(\begin{array}{c}
x_{1} \\
x_{2} \\
\ldots \\
x_{n}
\end{array}\right)=\left(\begin{array}{c}
0 \\
0 \\
\ldots \\
0
\end{array}\right)
$$

(order of $x_{1}, x_{2}, \ldots$ might have changed after elimination). By Gauss elimination property there exists such nonsingular square matrix $M$ for which $A^{\prime \prime}=M A^{\prime}$. Notice that this equality would still hold if we keep only first $m$ columns of $A^{\prime \prime}$ and $A^{\prime}$. Therefore, if $E$ and $D^{\prime}$ are corresponding square matrices, then equality $E=M D^{\prime}$ ( $E$ is the identity matrix) holds. Clearly, $M=\left(D^{\prime}\right)^{-1}$. It is known that

$$
M=\left(D^{\prime}\right)^{-1}=\left(\begin{array}{ccc}
\frac{\operatorname{det}\left(D_{1,1}^{\prime}\right)}{\operatorname{det}\left(D^{\prime}\right)} & \ldots & \frac{\operatorname{det}\left(D_{1, m}^{\prime}\right)}{\operatorname{det}\left(D^{\prime}\right)} \\
\ldots & \ldots & \ldots \\
\frac{\operatorname{det}\left(D_{m, 1}^{\prime}\right)}{\operatorname{det}\left(D^{\prime}\right)} & \ldots & \frac{\operatorname{det}\left(D_{m, m}^{\prime}\right)}{\operatorname{det}\left(D^{\prime}\right)}
\end{array}\right)
$$


where $D_{i, j}^{\prime}$ are adjoint matrices. Thus, $\left\|\operatorname{det} D^{\prime} \times M\right\|_{\infty}$ does not exceed the absolute value of determinant of matrix consisting of elements $1,-2,0$, (with at most two -1 and at most one 2 in each row), which we can bound by $\left(\sqrt{1^{2}+1^{2}+(-2)^{2}}\right)^{m}=6^{m / 2}$ by Hadamard inequality.

Since $A^{\prime}$ also consists of elements $-2,1,0$, equality $A^{\prime \prime}=M A^{\prime}$ implies that all elements of $\operatorname{det} D^{\prime} A^{\prime \prime}$ are integers with absolute values not exceeding $2 m 6^{m / 2} \leqslant 2 n 6^{n / 2}$.

Now we turn to construction of desired $y=\left(y_{1}, \ldots, y_{n}\right)$, satisfying all the conditions above. Let us consider equation $A^{\prime \prime} x=0$ and denote its first $m$ elements by $w_{1}, \ldots, w_{m}$, and remaining by $z_{1}, \cdots, z_{t}$, $m+t=n$. We have:

$$
A^{\prime \prime} x=0 \Leftrightarrow\left(\begin{array}{cccc}
1 & 0 & 0 & \ldots \\
0 & 1 & 0 & \ldots \\
0 & 0 & 1 & \ldots
\end{array}\right)\left(\begin{array}{c}
w_{1} \\
\cdots \\
w_{m} \\
z_{1} \\
\ldots \\
z_{t}
\end{array}\right)=\left(\begin{array}{c}
0 \\
0 \\
\ldots \\
0
\end{array}\right)
$$

or

$$
\begin{gathered}
w_{1}+a_{1,1} z_{1}+a_{1,2} z_{2}+\cdots+a_{1, t} z_{t}=0, \\
\ldots \\
w_{m}+a_{m, 1} z_{1}+a_{m, 2} z_{2}+\cdots+a_{m, t} z_{t}=0 .
\end{gathered}
$$

We know that any $a_{i, j}$ becomes integer when multiplied by $\operatorname{det} D^{\prime}$ not exceeding $2 n 6^{n / 2}$ by absolute value. From here we obtain that for any $w_{i}$ there exists such $\alpha_{i, 1}, \alpha_{i, 2}, \ldots, \alpha_{i, t}$ (negative correspondent elements of $A^{\prime \prime}$, multiplied by $\operatorname{det} D^{\prime}$ ), such that

$$
w_{i}=\frac{\alpha_{i, 1} z_{1}+\ldots+\alpha_{i, t} z_{t}}{\operatorname{det} D^{\prime}}
$$

where all of $\alpha_{i, j}$ are integer and bounded by $2 n 6^{n / 2}$ in absolute value.

We now aim to find such a solution $w_{1}, \cdots, w_{m}, z_{1}, \cdots, z_{t}$, where all variables are distinct, natural and do not exceed $4 n^{4} 6^{n / 2}$.

Now we demonstrate that it is possible to choose from multidimensional cube $[0, K-1]^{t}$, (where $\left.K=n^{2}\right)$, such $t$-tuple $\left(z_{1}, \ldots, z_{t}\right)$, so that all elements in $y=\left(z_{1}, \ldots, z_{t}, w_{1}, \ldots, w_{m}\right)$ would be distinct. Indeed, amount of possible points belonging to cube is $K^{t}$. Any equality of the form $z_{i}=z_{j}, z_{i}=$ $w_{j}, w_{i}=w_{j}$ determines a hyperplane of the form $\alpha_{1} z_{1}+\ldots+\alpha_{t} z_{t}=0$ - clearly, all integer points belonging to hyperplane can be projected onto the face of hypercube (and projections are integers, too). Therefore there are at most $K^{t-1}$ integer points on any hyperplane. In total, there are at most $C_{n}^{2}$ such hyperplanes, therefore, they contain at $\operatorname{most}_{n}^{2} K^{t-1}<K^{t}$ points in total.

Having this coordinates $\left(z_{1}, \ldots, z_{t}\right)$ we construct corresponding $w_{1}, \ldots, w_{m}$, multiply all elements of $y=\left(z_{1}, \cdots, w_{1}, \cdots\right)$ by $\operatorname{det} D^{\prime}$ and obtain an integer-valued vector, whose maximal element does not exceed either $n^{2} \times \operatorname{det} D^{\prime} \leqslant n^{2} \times 6^{n / 2}$, (if it was one of $z_{i}$ ), or $n \times \max (\alpha) \times \max \left(z_{i}\right) \leqslant n \times 2 n 6^{n / 2} \times n^{2}=$ $2 n^{4} 6^{n / 2}$ (if it was one of $w_{i}$ ) - and therefore we can bound maximal element as $2 n^{4} 6^{n / 2}$. To get rid off negative numbers, we shift coordinates of $y$ 'to right' to obtain set of naturals, with maximal element not exceeding $2 \times 2 n^{4} 6^{n / 2}$.

Remark 1. Clearly, one cannot get rid off exponential multiplier $c^{n}$, since there is not such compression for set $\left\{0,1,2,4, \ldots, 2^{n}\right\}$ that would make maximal element less than $2^{n}$. 
Lemma 2.2 (on compression into subset of segment of cubic length). If set $X$ of size $n$ belongs to segment $[1, \cdots, M]$, where $M=4 n^{4} 6^{n / 2}$, then there exists such subset $X^{\prime} \subseteq X,\left|X^{\prime}\right| \geqslant$ $|X| / 2$ which might be compressed into subset of segment $\left[n^{3}\right]$

Proof. Let us consider first prime numbers $p_{1}=2, p_{2}=3, p_{3}=5, \cdots$. Let us take the minimal prime number which does not divide any difference in $X$ and denote it by $p_{k+1}$. Then for any $p_{t}, t \leqslant k$, there are such distinct $x_{i}, x_{j}$, such that $p_{t} \mid\left(x_{i}-x_{j}\right)$. From here we obtain

$$
2 \times 3 \times 5 \times \cdots \times p_{k} \mid \prod_{i \neq j}\left(x_{i}-x_{j}\right)
$$

From here we obtain the following bound (via using $p_{k}>k,\left|x_{i}-x_{j}\right|<M$ ):

$$
2 \times 3 \times \cdots \times k \leqslant M^{\frac{n^{2}-n}{2}} .
$$

Apply log to both parts:

$$
k \ln k-k \leqslant \frac{n^{2}-n}{2} \ln M,
$$

from where it is easy to observe that $p_{k+1}<2 n^{3}$ for large enough $n$.

Thus, there exists such prime $p \leqslant 2 n^{3}$ which does not divide any difference in $X$. Let us now consider a set $X^{\prime}=\left\{x_{1}(\bmod p), x_{2}(\bmod p), \cdots\right\}$. It has size $n$, and belongs to segment $[0, \ldots, p-1]$, therefore intersects by half with one of the segments $L_{1}=[0, \cdots, p / 2]$, and $L_{2}=[p / 2, p-1]$ (it is clear, that if elements form a progression in $X$, then so their images do in $X^{\prime} \cap L_{i}$, provided that all of them belong to this image), and therefore we can remove at most half of the elements such that remaining set is compressible into subset of segment $\left[n^{3}\right]$.

Lemma 2.3 (on compression into subset of segment of almost-linear length). If set $X$ of size $n$ belongs to segment $\left[8 n^{3}\right]$, then for any $\epsilon>0$ there exists $X^{\prime} \subseteq X,\left|X^{\prime}\right| \geqslant(1 / 2-\epsilon)|X|$ such that $\left[C_{\epsilon} n \ln n\right]$, where $C_{\epsilon}$ is a constant, depending only on $\epsilon$.

Proof. For $n$ sufficiently large we consider prime numbers in segment $[2 n, \ldots, 2 c n \ln n]$, where $c$ is a positive constant. By Tchebyshev theorem, when $n$ is large enough, this segment would contain at least $c n$ prime numbers. We number them as $p_{1}, p_{2}, \cdots, p_{s}, s>c n$. Consider triples $(i, j, t)$, where $i, j, t$ are such that $p_{t} \mid\left(x_{i}-x_{j}\right)$. Notice that each pair $(i, j)$ of indexes participates in at most 2 triples, since $\left|x_{i}-x_{j}\right|<8 n^{3}$ and cannot be divisible by 3 or more distinct prime numbers exceeding $2 n$. Therefore, there are at most $n^{2}$ such triples. By Dirichlet's box principle some $p_{t}$ corresponds to at most $n^{2} / c n=n / c$ triples. We remove from $X$ all $x_{i}, x_{j}$, belonging to any of this triples, and remaining set $X_{r}$ would have size at least $\left(1-\frac{2}{c}\right)|X|$.

For set $X_{r}$ it is true that difference of any two distinct elements is not divisible by any prime $p_{t}<2 c n \ln n$, and in the same spirit as in previous lemma we remove from $X_{r}$ at most half of the elements such that remaining set might be compressed into subset $X^{\prime}$ of segment $[2 c n \ln n]$. Since we can take constant $c$ arbitrary large (and, accordingly, take $n>n(c)$ ), we have proved the desired assertion for any $\epsilon>0$.

Now we turn to a proof of the Hypothesis 1 in the special case $\epsilon \in(3 / 4,1)$ : 
Proof. We assume that $\epsilon \in(3 / 4,1)$. First we compress set $X$ of $n$ elements into subset of segment $\left[4 n^{4} 6^{n / 2}\right]$ by Lemma 2.1. Then we throw away at most half of the elements and compress $X$ into subset of segment $\left[n^{3}\right]$ by Lemma 2.2. Now we fix some $\delta>0$ and apply Lemma 2.3 to $X \subseteq$ $\left[1, \ldots, n^{3}\right] \sim\left[1, \ldots 8\left(\frac{n}{2}\right)^{3}\right]$, throw away at most $\left(\frac{1}{2}+\delta\right) \frac{n}{2}$ elements and compress remaining elements into the segment $\left[1, C_{\delta} \frac{n}{2} \ln \frac{n}{2}\right]$. In total we loose at most

$$
\frac{n}{2}+\left(\frac{1}{2}+\delta\right) \frac{n}{2}=\left(\frac{3}{4}+\frac{\delta}{2}\right) n
$$

elements, so we take $\delta$ such that inequality $\frac{3}{4}+\frac{\delta}{2} \leqslant \epsilon$ holds. Obviously, $\delta:=2 \epsilon-\frac{3}{2}>0$ would work.

\section{Proof of Theorem 1}

In what follows, we would need a following lemma:

Lemma 3.1 (on lower-bound for density). For any natural $a, b$ and natural $k \geqslant 3$ the following inequality holds:

$$
\rho_{k}(3 a b) \geqslant \rho_{3}(a) \rho_{k}(b) / 3 .
$$

Proof. Let us bisect a segment of length $3 a b$ into $a$ segments of length $3 b$. Let us choose among them those, whose numbers correspond to maximal subset of segment $[a]$, free of arithmetical progressions of length 3 (clearly, there would be exactly $g_{3}(a)=a \rho_{3}(a)$ of such segments). We bisect chosen segments into subsegments of length $b$, and only keep 'middle' ones. Then we take a maximal subset free of arithmetical progressions of length $k$ of size $g_{k}(b)=b \rho_{k}(b)$ in each of these middle subsegments. Clearly, the union of all those subsets does not contain any arithmetical progression of legnth $k$, and therefore $\rho_{k}(3 a b) \geqslant g_{3}(a) g_{k}(b) / 3 a b=\rho_{3}(a) \rho_{k}(b) / 3$.

Before proving Theorem 1, we need following inequality:

Lemma 3.2. For large enough natural $n$, natural $k \geqslant 3$ and positive real $\alpha \in(0,1 / 4)$, the following inequality holds:

$$
\phi_{k}(n)>\alpha n \rho_{k}\left(C_{\alpha, k} n \ln n\right) .
$$

Proof. Let us consider an arbitrary set $X$ of $n$ elements. By special case of Hypothesis 1 with $1-\frac{1+\alpha}{2} \rightarrow \epsilon$, one can remove at most $\epsilon n$ elements in such a way, so that remaining set might be compressed into subset $A$ of segment $\left[C_{\alpha} n \ln n\right]$ of size $\frac{1 / 4+\alpha}{2} n$. Let us set $m:=C_{\alpha} n \ln n$. Now we consider $\epsilon>0$ such that $\frac{1 / 4+\alpha}{2}(1-\epsilon)>\alpha$. Let us show that there exists such natural number $s$, depending only on $\alpha$, with the following property: if one considers maximal subset free of arithmetical progressions of length $k$ (which we denote by $T$ ) in the segment $[m+1, m+(s+1) m]$, then there is such a shift $A+x$ of set $A$, which has large intersection with $T$ (clearly, $|T|=(s+1) m \rho_{k}((s+1) m)$ ):

$$
|(A+x) \cap T| \geqslant(1-\epsilon)|A| \rho_{k}((s+1) m) .
$$

Indeed, let us consider shifts of $A$ 'to the right': $A+1, A+2, \ldots, A+s m$. Notice that any element of $T$, located left to $m+s m$, belongs to exactly $|A|$ shifts. Let $T_{1}:=T \cap[m+1, m+s m]$ and $T_{2}:=T \cap[m+s m+1, m+(s+1) m]$. Clearly $|T|=\left|T_{1}\right|+\left|T_{2}\right|$. Let us assume that (1) does not 
hold. By Dirichlet's box principle some shift of $A$ intersects $T$ by at least $\left|T_{1}\right||A| /$ sm elements, and therefore one can conclude that $\left|T_{1}\right||A| / s m \leqslant(1-\epsilon)|A| \rho_{k}((s+1) m)$, and therefore $\left|T_{2}\right| \geqslant|T|-\left|T_{1}\right| \geqslant$ $(1+s \epsilon) \rho_{k}((s+1) m)$ elements of $T$.

By Lemma 3.1 (we assume that $s+1$ is divisible by 3$)$ we see $\rho_{k}(m) \geqslant(1+s \epsilon) \rho_{k}((s+1) m) \geqslant$ $(1+s \epsilon) \rho_{3}((s+1) / 3) \rho_{k}(m)$ (we derive leftmost inequality from the fact that set free of progressions of length $k$ cannot have density more than $\rho_{k}(m)$ on segment of length $m$ ). Therefore, to get a contradiction, it is enough to take $s$ to be that large so that inequality $(1+s \epsilon) \rho_{3}((s+1) / 3) \geqslant 1$ holds. This is possible since $\rho_{3}(n) \geqslant \frac{1}{e^{c_{3} \sqrt{\ln n}}}$, denominator is subpolynomial, and the function $(1+s \epsilon) \rho_{3}\left(\frac{s+1}{3}\right)$ has polynomial growth on $s$. So, we obtained required $s$ depending on $\epsilon$ and $k$, or on $\alpha$ and $k$. So, now we have desired inequality $\phi_{k}(n)>\frac{1 / 4+\alpha}{2}(1-\epsilon) n \rho_{k}((s+1) m)>\alpha n \rho_{k}\left(H_{\alpha, k} n \ln n\right)$.

Now we turn to Theorem 1:

Proof. Let us suppose that statement of Theorem 1 does not hold for some $k \geqslant 3$. Therefore, there exists some $\epsilon>0$, such that for any $o(1)$ there is some segment $I=\left[m, m e^{(\ln m)^{1 / 2+o(1)}}\right]$, such that for any $n \in I$ inequality $\phi_{k}(n)<(1 / 4-\epsilon) g_{k}(n)$ holds. On the other side, by Lemma 3.2, any $n \in I$ satisfies $(1 / 4-\epsilon) g_{k}(n) \geqslant \alpha n \rho_{k}\left(C_{\alpha, k} n \ln n\right)$, where $\alpha>(1 / 4-\epsilon)$ (one can set $\left.\alpha:=1 / 4-\epsilon / 2\right)$. From here we obtain that for some constant $c>1\left(c:=\frac{\alpha}{1 / 4-\epsilon}\right)$ inequality $\rho_{k}(n)>c \rho(C n \ln n)$ takes place whenever $n \in I$.

Now we build the sequence $t_{1}=m, t_{2}=C t_{1} \ln t_{1}, t_{3}=C t_{2} \ln t_{2}, \cdots$ (we continue while $t_{i} \in I$ holds - clearly, there are at least $(\ln m)^{1 / 2+o(1)}$ such $\left.t_{i}\right)$.

Therefore,

$$
\rho_{k}\left(t_{1}\right)>c \rho_{k}\left(t_{2}\right)>c^{2} \rho_{k}\left(t_{3}\right)>\cdots
$$

Now, combining lower bound for $\rho_{k}(n)$, and the fact that sequence of $t_{i}$ has at least $(\ln m)^{1 / 2+o(1)}$ elements, the bound $\rho_{k}\left(t_{1}\right) \geqslant c^{i-1} \rho_{k}\left(t_{i}\right)$ would yield a contradiction for the last $t_{i}$ in the list.

\section{References}

[Beh46] F. A. Behrend. "On Sets of Integers Which Contain No Three Terms in Arithmetical Progression". Proceedings of the National Academy of Sciences 32.12 (1946), pp. 331-332. DOI: 10.1073/pnas.32.12.331. URL: https://www.pnas.org/content/32/12/331.

[Blo12] Thomas F. Bloom. "Translation invariant equations and the method of Sanders". Bulletin of the London Mathematical Society 44.5 (2012), pp. 1050-1067. DOI: 10.1112/blms / bds045. URL: https://londmathsoc . onlinelibrary.wiley .com/doi/abs/10.1112/ $\mathrm{blms} / \mathrm{bds} 045$.

[Gow01] W. T. Gowers. "A new proof of Szemerédi's theorem". Geom. Funct. Anal. 11.3 (2001), 465-588 (2001), erratum 11, no. 4, 869. DoI: https://doi.org/10.1007/s00039-0010332-9.

[KSS75] J. Komlós, M. Sulyok, and E. Szemerédi. "Linear problems in combinatorial number theory". Acta Mathematica Academiae Scientiarum Hungarica 26 (1975), pp. 113-121. DOI: https://doi.org/10.1007/BF01895954.

[OBr13] K. O’Bryant. "Thick Subsets that Do Not Contain Arithmetic Progressions". Integers 13 (2013), A18. DOI: 10.1515/9783110298161.249. arXiv: 0912.1494 [math.NT]. 
[Shk06] Ilya Shkredov. "Szemerédi's theorem and problems on arithmetic progressions". Uspekhi Mat. Nauk 61 (Jan. 2006), pp. 111-178. DOI: 10.4213/rm5293.

[TV06] T. Tao and V. Vu. "Additive Combinatorics". Vol. 105. Cambridge Univ. Press, Cambridge, 2006.

\author{
A.S. Semchankau \\ The Steklov Institute of Mathematics \\ 119991, Russian Federation, Moscow, Ulitsa Gubkina, 8 \\ aliaksei.semchankau@gmail.com
}

